\title{
Fox Grape cv. Bordô (Vitis labrusca L.) and Grapevine cv. Chardonnay (Vitis vinifera L.) Cultivated in vitro under Different Carbohydrates, Amino Acids and 6- Benzylaminopurine Levels
}

\author{
Dayse Cristina de Carvalho $^{1}$, André Luís Lopes da Silva ${ }^{2 *}$, Mariane Ruzza Schuck ${ }^{1}$, \\ Marivel Purcino $^{1}$, Guilherme Nakao Tanno ${ }^{1}$ and Luiz Antonio Biasi ${ }^{1}$ \\ ${ }^{1}$ Laboratório de Micropropagação de Plantas; Departamento de Fitotecnia e Fitossanitarismo; Setor de Ciências \\ Agrárias; Universidade Federal do Paraná; Curitiba - PR - Brasil. ${ }^{2}$ Laboratório de Processos Biotecnológicos; \\ Departamento de Engenharia Química; Universidade Federal do Paraná; Curitiba - PR - Brasil
}

\begin{abstract}
The aim of this work was to study the influence of sucrose and glucose, amino acids and BAP (6Benzylaminopurine) levels on in vitro shoot regeneration of fox grape cv. Bordô and grapevine cv. Chardonnay. The nodal segments from micropropagated material were used as explants and half-strength MS medium as the basal medium. Sucrose and glucose at 15, 30 and 45 g. $L^{-1}$ were tested as a carbon source and the supplementation of adenine, asparagine, alanine, glycine, cysteine, glutamine, arginine was tested at 40 g. $L^{-1 .}$ The BAP levels (1 and 5 $\mu M)$ in solid and double-phase media were evaluated and compared with a control medium without BAP. Bordô had best in vitro growth than Chardonnay. Sucrose was a better carbohydrate source than glucose for both the cultivars. Bordô and Chardonnay had different amino acid preferences for some parameters. In conclusion, for in vitro shoot regeneration from the nodal segments, culture on solid medium with $5 \mu M$ BAP, 15 g.L $L^{-1}$ sucrose for Bordô and 45 g. $L^{-1}$ sucrose for Chardonnay showed better results. Similarly, the supplementation of 40 g. $L^{-1}$ arginine for Bordô and 40 g.L.-1 arginine or glycine for Chardonnay showed better results.
\end{abstract}

Key words: Arginine, nodal segment, glucose, adenine, glycine

\section{INTRODUCTION}

The fox grape cv. Bordô is a fruit crop with easy adaptation to the variability of soil and climate conditions, good productivity, longevity and relative rusticity. Bordô fruits can be used to prepare red wine, juice, vinegar, and jellies and can also be consumed in natural form. The grapevine cv. Chardonnay is a green-skinned grape variety used to make white wine. Chardonnay is relatively easy to cultivate and can adapt to different conditions (Robinson 1999).
Nevertheless, grapevine culture shows several problems, mainly with the pests and diseases (Basso et al. 2010; Botelho et al. 2010).

Conventional genetic breeding is used to solve several problems but has some limitations. Abiotic and biotic stress can be totally or partially overcome via genetic transformation. Viral resistance incorporated via genetic transformation is becoming increasingly important and is likely to provide more effective strategies in the future (Rodrigues et al. 2009). The development of micropropagation protocols, somatic

*Author for correspondence: biasi@ufpr.br 
embryogenesis and cell suspension cultures represent the possibility to overcome the difficulties found in the current system of grapevines propagation. Moreover, these techniques allow the support for genetic breeding via biotechnological approaches such as somatic hybridization and genetic transformation, which are dependent on efficient protocols for plant regeneration (Carvalho et al. 2011).

The clonal propagation is the favorite method in many parts of the world, because it allows the production of more uniform offsprings (Radmann et al. 2009). The optimization of in vitro conditions is necessary to increase the multiplication in large-scale using bioreactors, which are more efficient than the conventional in vitro culture (Scheidt et al. 2009). However, many factors are involved for the success of an efficient micropropagation protocol. Plant mass propagation is extremely necessary to meet the demands of grapevine matrix plants of the proved genetic quality and health (Ayub et al. 2010; Schuck et al. 2012).

In tissue culture, carbohydrate sources play an important role as a source of energy and for maintaining the osmotic potential (Cuenca and Vieitez 2000). The nitrogen constituent of the amino acids and proteins is an essential factor for the development of the plant. In tissue culture, this element has significant effects in the cellular division and morphology (Kirby et al. 1987). Organic nitrogen is very important for the cellular growth and amino acids have been used as the source of nitrogen in the culture medium (Behrend and Mateles 1975). Moreover, the cultivars of grapevines demonstrate varied behavior in tissue culture, requiring specific studies for each cultivar, mainly when different species are used; therefore, the aim of this work was to study the influence of sucrose and glucose, different amino acids and BAP (6-benzylaminopurine) levels in solid and double-phase media on in vitro regeneration of fox grape cv. Bordô (Vitis labrusca) and grapevine cv. Chardonnay (Vitis vinifera).

\section{MATERIALS AND METHODS}

\section{In vitro establishment}

The plantlets of Vitis vinifera cv. Chardonnay and Vitis labrusca $\mathrm{cv}$. Bordô were established in vitro through the nodal segments from the shoot cuttings cultured in growth room and sub-cultured each 30 days on half strength salts and vitamins of MS medium (Murashige and Skoog 1962) according to Goussard (1981). These plantlets established in vitro were explant donors (fifth subculture), which were nodal segments (i.e. one centimeter height, without leaves and with one bud).

\section{Carbohydrates}

A three-way $(2 \times 2 \times 3)$ analysis of variance (ANOVA) experiment was carried out. Factor A consisted of two cultivars (cv. Bordô and Chardonnay), factor B consisted of two sources of carbohydrates (sucrose and glucose) and factor C consisted of carbohydrate levels $(15,30$ and 45 g. $\left.\mathrm{L}^{-1}\right)$. Basal medium was half strength $\mathrm{MS}$ medium solidified with 6 g.L. $\mathrm{L}^{-1}$ agar. Oxidation (\%), percentage of explants forming new shoots $(\%)$, rooting $(\%)$, shoot length $(\mathrm{cm})$, leaf number per shoot, root number per shoot, root length $(\mathrm{cm})$ and fresh weight $(\mathrm{mg})$ were evaluated after 60 days of in vitro culture. There was no subculture during the treatments.

Amino acids: A two-way (2x8) ANOVA experiment was carried out. Factor A consisted of two cultivars (cv. Bordô and Chardonnay) and factor B consisted of 40 g. $\mathrm{L}^{-1}$ of different amino acids (adenine, asparagine, alanine, glycine, cysteine, glutamine, arginine) and a control. The basal medium was half strength MS medium, supplemented with 30 g. $\mathrm{L}^{-1}$ sucrose and solidified with 6 g.L. ${ }^{-1}$ agar. Oxidation (\%), percentage of explants forming new shoots $(\%)$, rooting (\%), shoot length $(\mathrm{cm})$, leaf number per shoot, root number per shoot, root length $(\mathrm{cm})$ and fresh weight $(\mathrm{mg})$ were evaluated after 60 days of in vitro culture. There was no subculture during treatments.

\section{6-Benzylaminopurine}

A three-way $(2 \times 2 \times 3)$ ANOVA experiment was carried out. Factor A consisted of two cultivars (cv. Bordô and Chardonnay), factor B of two consistencies of medium (solid and double-phase) and factor C of BAP levels, which were 0,1 and 5 $\mu \mathrm{M}$. Basal medium consisted of half strength MS medium and 30 g.L $L^{-1}$ sucrose. For the solid phase, $30 \mathrm{~mL}$ medium solidified with 6 g.L $\mathrm{L}^{-1}$ agar were used and for the liquid phase, $4.0 \mathrm{~mL}$ medium was used. Oxidation (\%), percentage of explants forming new shoots (\%), callus formation at the base of the stem (\%), hyperhydricity (\%), shoot number per shoot and fresh weight $(\mathrm{mg})$ were 
evaluated after 60 days of in vitro culture. There was no subculture during the treatments.

\section{Culture conditions and statistical analysis}

All the media had their $\mathrm{pH}$ adjusted to 5.8 and were autoclaved at $1 \mathrm{~atm}$ and $121^{\circ} \mathrm{C}$ for $20 \mathrm{~min}$. The cultures were kept at $25 \pm 2^{\circ} \mathrm{C}$ under white fluorescent light $\left(28 \mu \mathrm{M} \mathrm{m}^{-2} \mathrm{~s}^{-1}\right)$ with a $16 \mathrm{~h}$ photoperiod. The experimental design was completely randomized in a factorial arrangement with four replicates of ten explants. The data was submitted to a normality analysis by the Lilliefors's method, followed by the ANOVA and by Tukey's test or Scott-Knott's test, both at a $\mathrm{P}<0.01$ and 0.05 . The variables from the counting were transformed to $\sqrt{x+0.5}$ and variables from the percentages were transformed to $\arcsin \sqrt{x / 100}$. All the statistical analyses were done following the procedures of the GENES software (Cruz 2001). All the experiments were repeated at least twice.

\section{RESULTS}

\section{Carbohydrates}

There was no interaction among the cultivar and carbohydrates for all the variables. Nevertheless, interaction among the cultivar and concentration was significant $(\mathrm{P}<0.01)$ for almost all the variables, with the exception of the root length. The rooting percentage was the only variable with interaction between the carbohydrate type and concentration $(\mathrm{B} \times \mathrm{C})$, and the interaction between the cultivar, carbohydrate type and concentration (A $\times$ B x C) was significant at $\mathrm{P}<0.01$ and $\mathrm{P}<0.05$, respectively (Table 1$)$.

Table 1 - Summary of the three-way ANOVA for the effect of carbohydrates (sucrose and glucose) on fox grape cv. Bordô (Vitis labrusca L.) and grapevine cv. Chardonnay (Vitis vinifera L.) nodal segments cultured in vitro. Data were evaluated after 60 days of in vitro culture. Oxidation (O \%), percentage of explants forming new shoots (S \%), rooting (R \%), shoot length (SL cm), leaf number per shoot (LS), root number per shoot (RS), root length (RL cm) and fresh weight (FW mg).

\begin{tabular}{|c|c|c|c|c|c|c|c|c|c|}
\hline \multirow[b]{2}{*}{ S.V. } & \multirow[b]{2}{*}{ d.f. } & \multicolumn{8}{|c|}{ Mean Square } \\
\hline & & $0 \%$ & $\mathrm{~S} \%$ & $\mathrm{R} \%$ & SL cm & $\mathbf{L S}$ & RS & RL cm & FW mg \\
\hline$A^{1}$ & 1 & $4009.2^{*}$ & $3099.4^{*}$ & $7849.1^{*}$ & $5.134^{*}$ & $3.456 *$ & $1.305^{*}$ & $4.530^{*}$ & $5934.3^{*}$ \\
\hline $\mathbf{B}^{2}$ & 1 & $192.9^{\mathrm{ns}}$ & $369.7^{\mathrm{ns}}$ & $1458.9^{*}$ & $0.709^{\mathrm{ns}}$ & $0.742^{* * *}$ & $0.160^{*}$ & $1.877^{*}$ & $490.6^{\mathrm{ns}}$ \\
\hline $\mathbf{C}^{3}$ & 2 & $80.5^{\mathrm{ns}}$ & $224.2^{\mathrm{ns}}$ & $585.6^{*}$ & $1.031^{*}$ & $0.602^{* *}$ & $0.168^{*}$ & $0.514^{\mathrm{ns}}$ & $732.5^{\text {** }}$ \\
\hline$A \times B$ & 1 & $549.1^{\mathrm{ns}}$ & $327.3^{\mathrm{ns}}$ & $278.1^{\mathrm{ns}}$ & $0.100^{\mathrm{ns}}$ & $0.113^{\mathrm{ns}}$ & $0.027^{\mathrm{ns}}$ & $0.557^{\mathrm{ns}}$ & $162.7^{\mathrm{ns}}$ \\
\hline $\mathbf{A} \times \mathbf{C}$ & 2 & $1179.1^{*}$ & $1525.1^{*}$ & $1143.3^{*}$ & $2.061^{*}$ & $1.775^{*}$ & $0.426^{*}$ & $0.471^{\mathrm{ns}}$ & $2519.6^{*}$ \\
\hline $\mathbf{B} \times \mathbf{C}$ & 2 & $245.5^{\mathrm{ns}}$ & $407.5^{\mathrm{ns}}$ & $610.8^{*}$ & $0.311^{\mathrm{ns}}$ & $0.265^{\mathrm{ns}}$ & $0.020^{\mathrm{ns}}$ & $0.114^{\mathrm{ns}}$ & $455.5^{\mathrm{ns}}$ \\
\hline$A \times B \times C$ & 2 & $42.8^{\mathrm{ns}}$ & $1.55^{\mathrm{ns}}$ & $366.4^{* * *}$ & $0.002^{\mathrm{ns}}$ & $0.007^{\mathrm{ns}}$ & $0.003^{\mathrm{ns}}$ & $0.001^{\mathrm{ns}}$ & $50.6^{\mathrm{ns}}$ \\
\hline Residual & 36 & 224.4 & 199.8 & 80.8 & 0.192 & 0.132 & 0.018 & 0.176 & 196.1 \\
\hline CV (\%) & & 23.9 & 53.8 & 49.1 & 74.6 & 27.7 & 14.8 & 58.4 & 73.1 \\
\hline
\end{tabular}

${ }^{1}$ Cultivar, ${ }^{2}$ Carbohydrate type, ${ }^{3}$ Carbohydrate concentration, ${ }^{*} \mathrm{P}<0.01,{ }^{* * *} \mathrm{P}<0.05,{ }^{\text {,s }}$ Not significant.

The cultivars presented statistical differences for the oxidation, shoot and rooting percentages, shoot length, leaf number per shoot, root number per shoot, root length and fresh weight. Bordô had better in vitro growth than Chardonnay cultivar for all variables (Table 2). Chardonnay tissues oxidized more than those of Bordô cultivar (Table 2 ). The results of oxidation percentage, shoot percentage, shoot length and fresh weight did not show differences in function of carbohydrate type and concentration (Table 1). However, sucrose promoted better results in root percentage, root number per shoot and root length than glucose for both the cultivars (Table 2). For the leaf number per shoot in Bordô cultivar, there was no statistical differences between sucrose and glucose (Table 2). The best result for rooting percentage was $50 \%$ in the medium containing $15 \mathrm{~g} . \mathrm{L}^{-1}$ glucose in $\mathrm{cv}$. Bordô. Glucose did not favor the rooting in cv. Chardonnay. Sucrose was more suitable than glucose for cv. Chardonnay; however the rooting percentage was lower (13.3\%). Bordô explants cultured on the media containing 15 or 30 g.L. sucrose had higher rooting rate (40 and $53.3 \%$, respectively) than on glucose containing media (Table 2). 
Table 2 - Effect of sucrose and glucose levels on fox grape cv. Bordô (Vitis labrusca L.) and grapevine cv. Chardonnay (Vitis vinifera L.) nodal segments after 60 days of in vitro culture.

\begin{tabular}{|c|c|c|c|c|c|c|}
\hline \multirow{2}{*}{$\begin{array}{l}\text { Oxidation (\%) } \\
\text { g.L } \text { L }^{-1}\end{array}$} & \multicolumn{3}{|c|}{ cv. Bordô } & \multicolumn{3}{|c|}{ cv. Chardonnay } \\
\hline & sucrose & glucose & Mean & sucrose & glucose & mean \\
\hline 15 & 56.6 & 56.6 & $56.6 \mathrm{~b}^{1}$ & 93.3 & 93.3 & $93.3 \mathrm{a}$ \\
\hline 30 & 56.6 & 60.0 & $58.3 \mathrm{~b}$ & 73.3 & 93.3 & $83.3 \mathrm{a}$ \\
\hline 45 & 83.3 & 73.3 & $78.3 \mathrm{a}$ & 70.0 & 86.6 & $78.3 \mathrm{a}$ \\
\hline Mean & & & $64.4 \mathrm{~B}^{2}$ & & & 84.9 A \\
\hline $\begin{array}{l}\text { Explants forming new shoots } \\
(\%)\end{array}$ & \multicolumn{3}{|c|}{ cv. Bordô } & \multicolumn{3}{|c|}{ cv. Chardonnay } \\
\hline g. $\mathbf{L}^{-1}$ & sucrose & glucose & Mean & sucrose & glucose & Mean \\
\hline 15 & 40.0 & 60.0 & $50.0 \mathrm{a}$ & 6.6 & 6.6 & $6.6 \mathrm{~b}$ \\
\hline 30 & 43.3 & 40.0 & $41.6 \mathrm{a}$ & 26.6 & 6.6 & $16.6 \mathrm{~b}$ \\
\hline 45 & 16.6 & 10.0 & $13.3 \mathrm{a}$ & 30.0 & 13.3 & $21.6 \mathrm{a}$ \\
\hline Mean & & & $34.9 \mathrm{~A}$ & & & 14.9 B \\
\hline Rooting (\%) & \multicolumn{3}{|c|}{ cv. Bordô } & \multicolumn{3}{|c|}{ cv. Chardonnay } \\
\hline g. $\mathrm{L}^{-1}$ & sucrose & glucose & Mean & sucrose & glucose & mean \\
\hline 15 & $40.0 \mathrm{aAB}$ & $50.0 \mathrm{aA}$ & & $0.0 \mathrm{aB}$ & $0.0 \mathrm{aB}$ & \\
\hline 30 & $53.3 \mathrm{aA}$ & $23.3 \mathrm{bB}$ & & $13.3 \mathrm{aBC}$ & $0.0 \mathrm{aC}$ & \\
\hline 45 & $26.6 \mathrm{bA}$ & $0.0 \mathrm{cB}$ & & $3.3 \mathrm{aB}$ & $3.3 \mathrm{aB}$ & \\
\hline \multicolumn{7}{|l|}{ Mean } \\
\hline Shoot length $(\mathrm{cm})$ & \multicolumn{3}{|c|}{ cv. Bordô } & \multicolumn{3}{|c|}{ cv. Chardonnay } \\
\hline g... $\mathrm{L}^{-1}$ & sucrose & glucose & Mean & sucrose & glucose & Mean \\
\hline 15 & 1.2 & 1.4 & $1.3 \mathrm{aA}$ & 0.1 & 0.1 & $0.1 \mathrm{aB}$ \\
\hline 30 & 1.3 & 1.0 & $1.1 \mathrm{aA}$ & 0.6 & 0.1 & $0.3 \mathrm{aB}$ \\
\hline 45 & 0.3 & 0.1 & $0.2 \mathrm{bA}$ & 0.6 & 0.2 & $0.4 \mathrm{aA}$ \\
\hline Mean & & & $0.8 \mathrm{~A}$ & & & $0.3 \mathrm{~B}$ \\
\hline Leaf number per explant & \multicolumn{3}{|c|}{ cv. Bordô } & \multicolumn{3}{|c|}{ cv. Chardonnay } \\
\hline g. $\mathrm{L}^{-1}$ & sucrose & glucose & Mean & sucrose & glucose & mean \\
\hline 15 & 3.1 & 3.7 & $3.4 \mathrm{aA}$ & 0.4 & 0.3 & $0.35 \mathrm{bB}$ \\
\hline 30 & 3.4 & 2.7 & $3.0 \mathrm{aA}$ & 1.4 & 0.1 & $0.75 \mathrm{aB}$ \\
\hline 45 & 0.8 & 0.2 & $0.5 \mathrm{bB}$ & 1.5 & 0.5 & $1.0 \mathrm{aA}$ \\
\hline Mean & $2.4 \mathrm{~A}$ & $2.2 \mathrm{~A}$ & $2.3 \mathrm{~A}$ & $1.1 \mathrm{~A}$ & $0.3 \mathrm{~B}$ & $0.7 \mathrm{~B}$ \\
\hline Root number per explant & \multicolumn{3}{|c|}{ cv. Bordô } & \multicolumn{3}{|c|}{ cv. Chardonnay } \\
\hline g. $\mathrm{L}^{-1}$ & sucrose & glucose & Mean & sucrose & glucose & mean \\
\hline 15 & 1.2 & 1.1 & $1.15 \mathrm{aA}$ & 0.0 & 0.0 & $0.00 \mathrm{bB}$ \\
\hline 30 & 1.2 & 0.7 & $0.95 \mathrm{aA}$ & 0.1 & 0.0 & $0.05 \mathrm{bB}$ \\
\hline 45 & 0.2 & 0.0 & $0.10 \mathrm{bA}$ & 0.3 & 0.1 & $0.20 \mathrm{aA}$ \\
\hline Mean & $0.86 \mathrm{~A}$ & $0.6 \mathrm{~B}$ & $0.73 \mathrm{~A}$ & $0.13 \mathrm{~A}$ & $0.03 \mathrm{~B}$ & 0.08 B \\
\hline Root length (cm) & \multicolumn{3}{|c|}{ cv. Bordô } & \multicolumn{3}{|c|}{ cv. Chardonnay } \\
\hline g. $\mathbf{L}^{-1}$ & sucrose & glucose & Mean & sucrose & glucose & mean \\
\hline 15 & 1.0 & 0.6 & & 0.0 & 0.0 & \\
\hline 30 & 1.3 & 0.6 & & 0.3 & 0.0 & \\
\hline 45 & 0.6 & 0.0 & & 0.2 & 0.0 & \\
\hline Mean & $0.9 \mathrm{~A}$ & $0.4 \mathrm{~B}$ & $0.7 \mathrm{~A}$ & $0.16 \mathrm{~A}$ & $0.0 \mathrm{~B}$ & 0.08 B \\
\hline Fresh weight (mg) & \multicolumn{3}{|c|}{ cv. Bordô } & \multicolumn{3}{|c|}{ cv. Chardonnay } \\
\hline g. $\mathbf{L}^{-1}$ & sucrose & glucose & Mean & sucrose & glucose & mean \\
\hline 15 & 37.1 & 47.2 & $42.1 \mathrm{aA}$ & 1.0 & 2.7 & $1.8 \mathrm{aB}$ \\
\hline 30 & 45.8 & 34.4 & $40.1 \mathrm{aA}$ & 12.9 & 1.7 & $7.3 \mathrm{aB}$ \\
\hline 45 & 11.4 & 4.6 & $8.0 \mathrm{bA}$ & 24.8 & 4.1 & $14.4 \mathrm{aA}$ \\
\hline Mean & & & 30.1 A & & & $7.8 \mathrm{~B}$ \\
\hline
\end{tabular}

${ }^{\mathrm{I}}$ Values with similar lower case letters in the same column and similar capital letters in the same line do not differ significantly $(\mathrm{P}<0.05)$ by Tukey's test. ${ }^{2}$ Values in bold with similar capital letters in the same line do not differ significantly $(\mathrm{P}<0.05)$ by Tukey's test. 
Carbohydrate levels results were statistically significant for the root percentage, length shoot, leaf number per shoot, root number per shoot and fresh weight (Table 1). Shoot length and fresh weight was higher at 15 and 30 g.. - $^{-1}$ in Bordô and there were no statistical differences in Chardonnay (Table 2). The leaf number per shoot was higher at 15 and 30 g.L $\mathrm{L}^{-1}$ in Bordô and at 30 and 45 g.L $\mathrm{L}^{-1}$ in Chardonnay (Table 2). Root number per shoot also presented higher values at 15 and $30 \mathrm{~g} . \mathrm{L}^{-1}$ in Bordô and at 45 g.L $\mathrm{L}^{-1}$ for Chardonnay.

\section{Amino acids}

The cultivars showed statistical differences for the leaf number per shoot, root number per shoot, fresh weight $(\mathrm{P}<0.01)$, oxidation and shoot percentages $(\mathrm{P}<0.05)$ but did not differ for the rooting percentage, shoot length and root length (Table 3). The amino acids presented statistical differences for the oxidation and shoot percentages $(\mathrm{P}<0.05)$, leaf number per shoot, root number per shoot, fresh weight, rooting percentage, shoot and root length $(\mathrm{P}<0.01)$. There was interaction among the cultivars and types of amino acids for the oxidation percentage, shoot percentage and root number per shoot $(\mathrm{P}<0.05)$; other variables did not show interaction (Table 3).

Arginine presented the best result for oxidation reduction in cv. Bordô (25\%) and glycine in cv. Chardonnay (37.5\%) (Table 4). Arginine promoted the highest shoot percentage in cv. Bordô $(75 \%)$ and glycine in cv. Chardonnay $(62.5 \%)$. On the other hand, alanine favored higher root number per shoot in cv. Bordô (2.7) and glycine and arginine in cv. Chardonnay (1.2 and 1.3, respectively) (Table 4). For both the cvs, glycine and arginine promoted larger rooting percentage (66 and $62.5 \%$, respectively). Higher shoot length was reached with asparagine, glycine, glutamine and arginine (varying from 1.5 to $2.4 \mathrm{~cm}$ ). Arginine allowed the largest leaf number per shoot (4.9) and cysteine, glutamine and arginine promoted the highest root length and fresh weight (Table 5).

Table 3 - Summary of two-way ANOVA for the effects of amino acids (adenine, asparagine, alanine, glycine, cysteine, glutamine and arginine) on fox grape cv. Bordô (Vitis labrusca L.) and grapevine cv. Chardonnay (Vitis vinifera $\mathrm{L}$.) nodal segments. Data evaluated after 60 days of in vitro culture. Oxidation (O \%), shoot (S \%), rooting (R \%), shoot length (SL cm), leaf number per shoot (LS), root number per shoot (RS), root length (RL cm) and fresh weight (FW mg).

\begin{tabular}{|c|c|c|c|c|c|c|c|c|c|}
\hline \multirow[b]{2}{*}{ S.V. } & \multirow[b]{2}{*}{ d.f. } & \multicolumn{8}{|c|}{ Mean Square } \\
\hline & & O \% & $\mathrm{S} \%$ & $\mathbf{R} \%$ & SL cm & $\mathbf{L S}$ & $\mathbf{R S}$ & RL cm & FW g \\
\hline $\mathrm{A}^{1}$ & 2 & $675.1^{* *}$ & $675.1^{* *}$ & $706.1^{\mathrm{ns}}$ & $0.638^{\mathrm{ns}}$ & $0.826^{*}$ & $0.849^{*}$ & $7.566^{\mathrm{ns}}$ & $0.013^{*}$ \\
\hline $\mathbf{B}^{2}$ & 7 & $379.7^{* *}$ & $379.7^{* *}$ & $1413.6^{*}$ & 3.420 * & $0.801 *$ & $0.393^{*}$ & 23.79 * & 0.011 * \\
\hline$A \times B$ & 7 & $323.7^{* *}$ & $323.7^{* *}$ & $404.4^{\mathrm{ns}}$ & $0.678^{\mathrm{ns}}$ & $0.302^{\mathrm{ns}}$ & $0.128^{* *}$ & $4.796^{\mathrm{ns}}$ & $0.002^{\mathrm{ns}}$ \\
\hline Residual & 59 & 142.8 & 142.8 & 227.2 & 0.475 & 0.163 & 0.046 & 2.430 & 0.001 \\
\hline CV $(\%)$ & & 24.3 & 29.2 & 37.5 & 49.7 & 23.0 & 17.4 & 76.6 & 56.0 \\
\hline
\end{tabular}

${ }^{\mathrm{T}}$ Cultivar, ${ }^{2}$ Amino acids, ${ }^{*} \mathrm{P}<0.01,{ }^{* *} \mathrm{P}<0.05,{ }^{\text {ns }}$ Not significant.

Table 4 - Effect of different amino acids on growth and rooting of nodal segments of Vitis labrusca cv. Bordô and Vitis vinifera cv. Chardonnay after 60 days of in vitro culture on half strength MS medium. Variables presented significant interaction between cultivars and types of amino acids.

\begin{tabular}{|c|c|c|c|c|c|c|}
\hline \multirow{2}{*}{ Amino acid (40 mg.L $\left.\mathrm{L}^{-1}\right)$} & \multicolumn{2}{|c|}{ Shoot $(\%)$} & \multicolumn{2}{|c|}{ Oxidação (\%) } & \multicolumn{2}{|c|}{ Root number per shoot } \\
\hline & Bordô & Chardonnay & Bordô & Chardonnay & Bordô & Chardonnay \\
\hline Control & $43.3 \mathrm{~b}^{1} \mathrm{~A}^{2}$ & $26.6 \mathrm{cB}$ & $56.6 \mathrm{bB}$ & $73.3 \mathrm{aA}$ & $1.26 \mathrm{cA}$ & $0.13 \mathrm{~dB}$ \\
\hline Adenine & $27.5 \mathrm{cB}$ & $45.0 \mathrm{bA}$ & $72.5 \mathrm{aA}$ & $57.5 \mathrm{aA}$ & $0.35 \mathrm{eA}$ & $0.25 \mathrm{dA}$ \\
\hline Asparagine & $45.0 \mathrm{bA}$ & $36.6 \mathrm{cA}$ & $55.0 \mathrm{bA}$ & $63.3 \mathrm{aA}$ & $1.52 \mathrm{cA}$ & $0.66 \mathrm{cB}$ \\
\hline Alanine & $50.0 \mathrm{bA}$ & $25.0 \mathrm{cB}$ & $50.0 \mathrm{bB}$ & $75.0 \mathrm{aA}$ & $2.77 \mathrm{aA}$ & $0.82 \mathrm{bB}$ \\
\hline Glycine & $45.0 \mathrm{bB}$ & $62.5 \mathrm{aA}$ & $55.0 \mathrm{bA}$ & $37.5 \mathrm{bB}$ & $1.27 \mathrm{cA}$ & $1.22 \mathrm{a} \mathrm{A}$ \\
\hline Cysteine & $55.0 \mathrm{bA}$ & $35.0 \mathrm{cB}$ & $45.0 \mathrm{bB}$ & $65.0 \mathrm{aA}$ & $2.00 \mathrm{bA}$ & $0.95 \mathrm{bB}$ \\
\hline Glutamine & $50.0 \mathrm{bA}$ & $32.5 \mathrm{cB}$ & $50.0 \mathrm{bB}$ & $67.5 \mathrm{aA}$ & $0.95 \mathrm{dA}$ & $0.65 \mathrm{cB}$ \\
\hline Arginine & $75.0 \mathrm{aA}$ & $45.0 \mathrm{bB}$ & $25.0 \mathrm{cB}$ & $55.0 \mathrm{aA}$ & $1.95 \mathrm{bA}$ & $1.30 \mathrm{aB}$ \\
\hline CV $(\%)$ & \multicolumn{2}{|c|}{29.2} & \multicolumn{2}{|c|}{24.3} & \multicolumn{2}{|c|}{17.4} \\
\hline
\end{tabular}

${ }^{1}$ Means within a column followed by the same lower case letter for each parameter are not different at $\mathrm{P}<0.05$ by Scott-Knott's test. ${ }^{2}$ Means within a line followed by the same capital letter for each parameter are not different at $\mathrm{P}<0.05$ by Scott-Knott's test. 
Table 5 - Effect of different amino acids on growth and rooting of nodal segments of Vitis labrusca cv. Bordô and Vitis vinifera cv. Chardonnay on half strength MS medium after 60 days of in vitro culture. Variables did not show interaction between cultivars and types of amino acids. The data are the mean values of both species.

\begin{tabular}{|c|c|c|c|c|c|}
\hline $\begin{array}{r}\text { Amino acid } \\
\left(40{\left.\mathrm{mg} . \mathrm{L}^{-1}\right)}^{2}\right.\end{array}$ & $\begin{array}{c}\text { Rooting } \\
(\%)\end{array}$ & $\begin{array}{c}\text { Shoot length } \\
(\mathrm{cm})\end{array}$ & $\begin{array}{c}\text { Leaf number per } \\
\text { shoot }\end{array}$ & $\begin{array}{c}\text { Root length } \\
(\mathrm{cm})\end{array}$ & $\begin{array}{c}\text { Fresh weight } \\
(\mathrm{mg})\end{array}$ \\
\hline Control & $33.3 c^{1}$ & $0.98 \mathrm{~b}$ & $2.4 \mathrm{c}$ & $0.84 \mathrm{~b}$ & $29 \mathrm{~b}$ \\
\hline Adenine & $11.2 \mathrm{~d}$ & $0.39 \mathrm{~b}$ & $1.1 \mathrm{~d}$ & $0.03 \mathrm{~b}$ & $22 \mathrm{~b}$ \\
\hline Asparagine & $40.4 \mathrm{~b}$ & $1.60 \mathrm{a}$ & $2.6 \mathrm{c}$ & $1.48 \mathrm{~b}$ & $56 \mathrm{~b}$ \\
\hline Alanine & $46.2 \mathrm{~b}$ & $1.22 \mathrm{~b}$ & $2.6 \mathrm{c}$ & $1.44 \mathrm{~b}$ & $57 \mathrm{~b}$ \\
\hline Glycine & $66.0 \mathrm{a}$ & $1.56 \mathrm{a}$ & $3.4 \mathrm{~b}$ & $1.50 \mathrm{~b}$ & $68 \mathrm{~b}$ \\
\hline Cysteine & $50.0 \mathrm{~b}$ & $1.27 \mathrm{~b}$ & $2.3 \mathrm{c}$ & $2.65 \mathrm{a}$ & $94 \mathrm{a}$ \\
\hline Glutamine & $50.0 \mathrm{~b}$ & $1.62 \mathrm{a}$ & $3.2 \mathrm{~b}$ & $3.55 \mathrm{a}$ & $89 a$ \\
\hline Arginine & $62.5 \mathrm{a}$ & $2.43 \mathrm{a}$ & $4.9 \mathrm{a}$ & $4.76 \mathrm{a}$ & $122 \mathrm{a}$ \\
\hline Mean & 44.9 & 1.38 & 2.8 & 2.03 & 67.2 \\
\hline CV(\%) & 37.5 & 49.7 & 23.0 & 76.6 & 56.0 \\
\hline
\end{tabular}

${ }^{1}$ Means within a column followed by the same letter for each parameter are not different at $\mathrm{P}<0.05$ by Scott-Knott's test.

\section{6-Benzylaminopurine}

The cultivars showed statistical differences for the oxidation, shoot, callus and hyperhydricity percentages $(\mathrm{P}<0.01)$. Consistency of the media presented statistical differences for the oxidation and shoot percentages $(\mathrm{P}<0.05)$, for callus and hyperhydricity percentages and fresh weight $(\mathrm{P}<0.01)$. The BAP levels showed statistical differences for the oxidation percentage, shoot percentage, callus percentage, hyperhydricity percentage and fresh weight $(\mathrm{P}<0.01)$. There was interaction among the cultivar and consistency of the media (A x B) for the oxidation, shoot, callus and hyperhydricity percentages $(\mathrm{P}<0.01)$. There was interaction among the cultivars and BAP level (A $\mathrm{x}$ C) for the hyperhydricity percentage and shoot number $(\mathrm{P}<0.01)$. There was interaction among the consistency of the media and BAP level $(\mathrm{B} \times \mathrm{C})$ for the callus percentage and fresh weight
$(\mathrm{P}<0.01)$. The triple interactions were significant for the callus percentage, hyperhydricity percentage and shoot number $(\mathrm{P}<0.01)$ (Table 6).

The Chardonnay showed higher oxidation rate than the Bordô. The presence of BAP decreased the oxidation percentage. Consistency of the media did not influence the oxidation percentage in $\mathrm{cv}$. Chardonnay; however, on solid medium, the oxidation percentage in cv. Bordô explants was inferior to the rate observed on double phase medium (Table 7). The Bordô presented higher shoot percentage (i.e., the percentage of explants forming new shoots) than Chardonnay. The presence of BAP increased the formation of new shoots. The consistency of the media did not influence the shoot percentage in the cv. Chardonnay; however, there was a higher formation of new shoots on solid medium than on double phase medium in the cv. Bordô.

Table 6 - Summary of three-way ANOVA for the effects of BAP (6-Benzylaminopurine) level on fox grape cv. Bordô (Vitis labrusca L.) and grapevine cv. Chardonnay (Vitis vinifera L.) nodal segments cultured on solid and double-phase media. Data evaluated after 60 days of in vitro culture. Oxidation (O \%), shoot (S \%), callus (C \%), hyperhydricity (H \%), shoot number (SN) and fresh weight (FW mg).

\begin{tabular}{|c|c|c|c|c|c|c|c|}
\hline \multirow[b]{2}{*}{ S.V. } & \multicolumn{7}{|c|}{ Mean Square } \\
\hline & d.f. & $0 \%$ & $\mathrm{~S} \%$ & C \% & Н \% & SN & FW mg \\
\hline$A^{1}$ & 1 & $4583.6^{*}$ & $4951.0^{*}$ & $11846.8^{*}$ & $9098.3^{*}$ & $0.0008^{\mathrm{ns}}$ & $8176.6^{\mathrm{ns}}$ \\
\hline $\mathrm{B}^{2}$ & 1 & $602.1^{* *}$ & $739.8^{\text {** }}$ & $3664.8^{*}$ & $588.0^{*}$ & $0.0140^{\mathrm{ns}}$ & $913206.9^{*}$ \\
\hline$C^{3}$ & 2 & $3955.2^{*}$ & $3802.3^{*}$ & $13002.2^{*}$ & $3300.8^{*}$ & $0.1073^{\mathrm{ns}}$ & $548888.6^{*}$ \\
\hline$A \times B$ & 1 & $2632.6^{*}$ & $2366.6^{*}$ & $3327.5^{*}$ & 4299.1 * & $0.0437^{\mathrm{ns}}$ & $29674.8^{\mathrm{ns}}$ \\
\hline $\mathrm{A} \times \mathrm{C}$ & 2 & $27.7^{\mathrm{ns}}$ & $17.39^{\mathrm{ns}}$ & $380.7^{\mathrm{ns}}$ & $2064.5^{*}$ & $1.6258^{*}$ & $18913.3^{\mathrm{ns}}$ \\
\hline $\mathrm{B} \times \mathrm{C}$ & 2 & $279.6^{\mathrm{ns}}$ & $329.3^{\mathrm{ns}}$ & $942.4^{*}$ & $118.5^{\mathrm{ns}}$ & $0.1624^{\mathrm{ns}}$ & $300187.2^{*}$ \\
\hline$A \times B \times C$ & 2 & $142.1^{\mathrm{ns}}$ & $205.0^{\mathrm{ns}}$ & $937.3^{*}$ & $1073.5^{*}$ & $1.0407^{*}$ & $3229.5^{\mathrm{ns}}$ \\
\hline Residual & 36 & 131.6 & 135.8 & 127.8 & 47.39 & 0.0835 & 7215.0 \\
\hline CV(\%) & & 41.3 & 18.8 & 23.3 & 34.9 & 19.6 & 35.9 \\
\hline
\end{tabular}

${ }^{1}$ Cultivar, ${ }^{2}$ Consistency of the medium, ${ }^{3} \mathrm{BAP}$ level, ${ }^{*} \mathrm{P}<0.01,{ }^{* *} \mathrm{P}<0.05,{ }^{\text {ns }}$ Not significant. 
Table 7 - Effect of BAP (6-Benzylaminopurine) levels on nodal segments from fox grape cv. Bordô (Vitis labrusca L.) and grapevine cv. Chardonnay (Vitis vinifera L.) cultured on solid and double-phase media after 60 days.

\begin{tabular}{|c|c|c|c|c|c|c|}
\hline \multirow{2}{*}{$\begin{array}{l}\text { Oxidation }(\%) \\
\text { BAP concentration }(\mu \mathrm{M})\end{array}$} & \multicolumn{3}{|c|}{ cv. Bordô } & \multicolumn{3}{|c|}{ cv. Chardonnay } \\
\hline & Solid & Double-phase & mean & Solid & Double-phase & mean \\
\hline 0 & 17.5 & 55.0 & $36.2 \mathrm{a}^{1}$ & 72.5 & 67.5 & $70 \mathrm{a}$ \\
\hline 1 & 0.0 & 17.5 & $8.7 \mathrm{~b}$ & 40.0 & 10.0 & $25 \mathrm{~b}$ \\
\hline 5 & 0.0 & 12.5 & $6.2 \mathrm{~b}$ & 25.0 & 25.0 & $25 \mathrm{~b}$ \\
\hline Mean & $5.8 \mathrm{~B}$ & $28.3 \mathrm{~A}$ & & $45.8 \mathrm{~A}$ & $34.2 \mathrm{~A}$ & \\
\hline \multirow[t]{2}{*}{ Shoot $(\%)$} & \multicolumn{3}{|c|}{ cv. Bordô } & \multicolumn{3}{|c|}{ cv. Chardonnay } \\
\hline & Solid & Double-phase & mean & Solid & Double-phase & mean \\
\hline $\mathbf{0}$ & 82.5 & 45 & $63.7 \mathrm{~b}$ & 27.5 & 32.5 & $30 \mathrm{~b}$ \\
\hline 1 & 100 & 82.5 & $91.2 \mathrm{a}$ & 60 & 90 & $75 \mathrm{a}$ \\
\hline 5 & 100 & 87.5 & $93.7 \mathrm{a}$ & 75 & 67.5 & $71.2 \mathrm{a}$ \\
\hline Mean & $94.2 \mathrm{~A}$ & $63.7 \mathrm{~B}$ & & $54.2 \mathrm{~A}$ & $63.3 \mathrm{~A}$ & \\
\hline \multirow[t]{2}{*}{ Callus (\%) } & \multicolumn{3}{|c|}{ cv. Bordô } & \multicolumn{3}{|c|}{ cv. Chardonnay } \\
\hline & Solid & Double-phase & mean & Solid & Double-phase & Mean \\
\hline $\mathbf{0}$ & $80 \mathrm{bA}$ & $0 \mathrm{bB}$ & & $0 \mathrm{cB}$ & $0 \mathrm{bB}$ & \\
\hline 1 & $100 \mathrm{aA}$ & $82.5 \mathrm{aA}$ & & $35 \mathrm{bB}$ & $55 \mathrm{aB}$ & \\
\hline 5 & $100 \mathrm{aA}$ & $87.5 \mathrm{aA}$ & & $80 \mathrm{aA}$ & $57.5 \mathrm{aB}$ & \\
\hline \multicolumn{7}{|l|}{ mean } \\
\hline \multirow[t]{2}{*}{ Hyperhydricity (\%) } & \multicolumn{3}{|c|}{ cv. Bordô } & \multicolumn{3}{|c|}{ cv. Chardonnay } \\
\hline & Solid & Double-phase & mean & Solid & Double-phase & Mean \\
\hline $\mathbf{0}$ & $0 \mathrm{bA}$ & $0 \mathrm{aA}$ & & $0 \mathrm{cA}$ & $10 \mathrm{bA}$ & \\
\hline 1 & $35 \mathrm{aB}$ & $0 \mathrm{aC}$ & & $15 \mathrm{bC}$ & $77.5 \mathrm{aA}$ & \\
\hline 5 & $0 \mathrm{bC}$ & $0 \mathrm{aC}$ & & $40 \mathrm{aB}$ & $80 \mathrm{aA}$ & \\
\hline \multicolumn{7}{|l|}{ mean } \\
\hline \multirow[t]{2}{*}{ Leaf number per explant } & \multicolumn{3}{|c|}{ cv. Bordô } & \multicolumn{3}{|c|}{ cv. Chardonnay } \\
\hline & Solid & Double-phase & mean & Solid & Double-phase & Mean \\
\hline $\mathbf{0}$ & $0.8 \mathrm{cA}$ & $0.4 \mathrm{bA}$ & & $0.3 \mathrm{cA}$ & $0.3 \mathrm{cA}$ & \\
\hline 1 & $1.8 \mathrm{bB}$ & $3.3 \mathrm{aA}$ & & $1.4 \mathrm{bB}$ & $3.5 \mathrm{bA}$ & \\
\hline 5 & $3.1 \mathrm{aBC}$ & $3.4 \mathrm{aAB}$ & & $2.8 \mathrm{aC}$ & $3.8 \mathrm{aA}$ & \\
\hline \multicolumn{7}{|l|}{ mean } \\
\hline \multirow[t]{2}{*}{ Fresh weight (mg) } & \multicolumn{3}{|c|}{ cv. Bordô } & \multicolumn{3}{|c|}{ cv. Chardonnay } \\
\hline & Solid & Double-phase & mean & Solid & Double-phase & Mean \\
\hline $\mathbf{0}$ & $93.6 \mathrm{bA}^{2}$ & $59.9 \mathrm{cA}$ & & $22.2 \mathrm{cA}$ & $37.6 \mathrm{cA}$ & \\
\hline 1 & $104.8 \mathrm{aB}$ & $323.7 \mathrm{bA}$ & & $59.6 \mathrm{bB}$ & $439.6 \mathrm{bA}$ & \\
\hline 5 & $132.2 \mathrm{aB}$ & $625.4 \mathrm{aA}$ & & $177.9 \mathrm{aB}$ & $759.2 \mathrm{aA}$ & \\
\hline mean & $110.2 \mathrm{~B}$ & $336.3 \mathrm{~A}$ & & $86.5 \mathrm{~B}$ & $412.1 \mathrm{~A}$ & \\
\hline
\end{tabular}

${ }^{\mathrm{T}}$ Values with similar lower case letters in the same column and similar capital letters in the same line do not differ significantly $(\mathrm{P}<0.05)$ by Tukey's test. ${ }^{2}$ Values with similar lower case letters in the same column and similar capital letters in the same line for interaction among BAP level and consistency of the media do not differ significantly $(\mathrm{P}<0.05)$ by Tukey's test.

The presence of the BAP increased the callogenesis in the cv. Bordô, but the consistency of the media and BAP levels did not influence it (varying from 82.5 to $100 \%$ ). Nevertheless, in the cv. Chardonnay, the most suitable BAP level for the calli percentage was $5 \mu \mathrm{M}$, reaching $80 \%$ in the solid medium. Even in the absence of BAP, the callus was formed in the cv. Bordô explants cultured on the solid medium $(80 \%)$ (Table 7$)$. In order to reduce the callogenesis occurrence in the cv. Bordô, it was possible that the highest BAP levels could decrease the callus percentage, as observed in the cv. Perlette where nodal explants cultivated on the MS supplemented with $10 \mu \mathrm{M}$ BAP promoted $50 \%$ callus comparing to $80 \%$ callus obtained in $5 \mu \mathrm{M}$ BAP. However, the formation of new shoots was not influenced (Jaskani et al. 2008). In the cv. Chardonnay, the callogenesis decreased by using $1 \mu \mathrm{M}$ BAP without alteration in the number of new shoots (Table 7).

The Chardonnay had higher tendency to hyperhydricity than the Bordô. Double-phase medium did not promote hyperhydricity in the 
Bordô. On the other hand, Chardonnay cultured on double-phase medium had the most elevated hyperhydricity percentage even in the absence of BAP (10\%). Hyperhydricity was also observed in the Chardonnay cultured on the solid medium. However, the percentage was lower than on double-phase medium. Hyperhydricity in the Bordô was just observed at $1 \mu \mathrm{M}$ BAP in solid medium.

The most suitable BAP concentration to obtain an elevated number of leaves per explant and high fresh weight was $5 \mu \mathrm{M}$ in double-phase system for the Chardonnay and Bordô, but the leaf number per explant did not differ on both types of the medium for cv. Bordô (Table 7). However, it has been shown that $2.5 \mu \mathrm{M}$ BAP was more efficient for the leaf number per explant in the nodal segments of cv. Bordô cultured on solid MS medium, but these nodal segments were introduced with a leaf (Ayub et al. 2010), while in the present work, the leaves were cut. Moreover, the medium used was double-phase half strength MS medium.

\section{DISCUSSION}

\section{Carbohydrates}

Sucrose is a non-reducing sugar. In this, the ketone or aldehyde group is reduced to an alcohol or these groups are combined with a similar group from other sugar. Glucose is a reducing sugar (i.e., sugars containing an aldehyde or ketone group exposed). Non-reducing sugars are less reactive than the reducing sugars. Therefore, non-reducing sugars are more translocated (Taiz and Zeiger 2004). In the tissue culture, shoot and root morphogenesis is strongly dependent on the carbohydrate supply. Sucrose is commonly used in the culture media for the morphogenesis; but a few reports have shown glucose as a better carbon source. However, glucose proved to be a regulatory factor in the root culture of Eucalyptus globulus where it induced up to $89 \%$ rooting (Corrêa et al. 2005) depending on what root formation stage it was applied.

Bordô seemed to be more sensitive than the Chardonnay to the osmotic stress as the growth parameters decreased at 45 g. $\mathrm{L}^{-1}$ carbohydrates. On the other hand, Chardonnay seemed to have more exigencies in terms of energy than the Bordô, while some growth parameters were favored at $45 \mathrm{~g} . \mathrm{L}^{-1}$ carbohydrates, such as root and leaf number per explant and shoot percentage (Table 2).

\section{Amino acids}

The preference for certain amino acid as the source of nitrogen can be associated to the genetic differences among the grapevine cultivars. The supplementation with amino acids can decrease or increase the oxidation in the nodal segments of grapevines. This result can be influenced by many factors, one of them could be due to the chemical nature of the amino acid. The amino acids can be grouped according to its R-group (i.e., $\mathrm{R}$ is an organic substituent); there are basic amino acids (e.g. arginine), acidic amino acids (e.g., glutamine and asparagine), aliphatic amino acids (e.g., glycine and alanine) and amino acids with a sulphur content side chain (e.g., cysteine) (Rossetti 1996). It was possible that arginine decreased the oxidation percentage in the cv. Bordô because it was precursor of putrescine (i.e., a polyamine) (Tiburcio et al. 1985). The putrescine decreases the oxidative damage and improves the plant regeneration by acting as plant growth substance (Tang et al. 2004). On the other hand, glycine decreased the oxidation percentage in the cv. Chardonnay, which could be associated to this amino acid as a precursor of nucleotides, which played central roles in the metabolism. Nucleotides serve as the sources of chemical energy (adenosine triphosphate and guanosine triphosphate), participate in the cellular signaling (cyclic guanosine monophosphate and cyclic adenosine monophosphate) and are incorporated into important cofactors of enzymatic reactions (coenzyme A, flavin adenine dinucleotide, flavin mononucleotide, and nicotinamide adenine dinucleotide phosphate) (Alberts et al. 2002).

The nitrogen from amino acids is assimilated more quickly by the carbonic skeletons during the metabolism and synthesis of proteins, when compared to the nitrogen from the inorganic sources (Carvalho et al. 2004). However, different amino acids have different functions in the plant metabolism. Glutamine and asparagine are sources of $\mathrm{N}$ frequently found in the phloem; asparagine also acts as an element key in the transport and in the storage of the nitrogen (Taiz and Zeiger 2004). Arginine is also a source of nitric oxide (Polacco 1977), which is an important molecule that acts in many tissues to regulate a diverse range of physiological processes (Palavan-Unsal and Arisan 2009). Cysteine is also a source of sulphate 
(Taiz and Zeiger 2004). Another effect of amino acids in the tissue culture is the synergic effect with the plant growth regulators, as found in pigeonpea and peanut. In the peanut, the highest frequency of plant regeneration was induced by the BAP in combination with IAA or IAA-1alanine, while in pigeonpea, the BAP in combination with IAA or IAA-l-aspartic acid produced best results (Eapen and George 1993).

\section{6-Benzylaminopurine}

The influence of BAP in the oxidation of the explants is dependent of the species or cultivar, besides the used concentration. In Melaleuca alternifolia, the increase in BAP level favored the oxidation in explants cultivated in the liquid and solid MS or WPM medium. The larger oxidation percentage for all the media was observed in 4.4 $\mu \mathrm{M}$ BAP (Oliveira et al. 2010). On the other hand, in Vitis vinifera cv. Merlot, the BAP levels did not influence the oxidation percentage (Carvalho et al. 2011). But meristems of grapevine cv. 'A Dona' cultured in the presence of 2 to $10 \mu \mathrm{M}$ BAP presented reduced percentage of oxidation (Passos et al. 1985). At the stage of in vitro multiplication, the capacity of the explants to survive, to develop and proliferate is the consequence of several factors, such as genetic, physiological factors and environmental conditions of culture (Kozai et al. 1997). BAP has a great influence in the shoot proliferation of grapevines, as observed in $\mathrm{cv}$. Merlot (Carvalho et al. 2011), cv. Bordô (Ayub et al. 2010) and grapevine rootstock cv. 'VR043-43' (Machado et al. 2007). The consistency of the culture medium also exercises fundamental effect on the morphogenesis and on the shoot growth (Karasawa et al. 2002). Nutrients and water present in the double-phase medium have largest ability to be absorbed, due to a greater amount of nutrients and water available; besides, there are no physical barriers in the culture medium, which increases the explant contact with the nutrients and water whereby it usually increases the multiplication rate.

Nodal segments of Vitis vinifera cv. Albariño cultivated in the double-phase medium supplemented with $8.8 \mu \mathrm{M}$ BAP had a two-fold increase of the multiplication rate when compared with the culture in solid medium (Couselo et al. 2006). This was different from the present results, where the use of the double-phase medium produced results (percentage of explants forming new shoots) similar to those obtained on solid medium for Chardonnay and inferior results for Bordô cv. These differences could be due to genetic characteristics of these cultivars; moreover, the BAP level was lower $(1$ and $5 \mu \mathrm{M})$ than used by Couselo et al. (2006) that was 8.8 $\mu \mathrm{M}$.

The double-phase medium promoted hyperhydricity in Chardonnay, which was also observed in cv. Albariño (Couselo et al. 2006). Hyperhydricity is a complex phenomenon that can provoke the death of the plant and can be caused by several factors, the main being the high relative humidity in the flask, especially when the medium is liquid (Abdoli et al. 2007; Haq and Dahot 2007; Scheidt et al. 2011; Silva et al. 2012). High BAP levels also induce hyperhydricity occurrence (Oliveira et al. 2010). The hyperhydricity in liquid culture can be controlled by osmoregulators such as maltose and sorbitol (Ziv 1989) and improved vessel aeration (Rossetto et al. 1992).

The capacity of calli formation promoted by the BAP in grapevine is already known (Jaskani et al. 2008). Similar results were obtained in the nodal segments of grapevine rootstock cv. 'Jales', where the BAP induced the calli formation, varying between 32.8 and $100 \%$, for the concentrations of 2.5 to $10 \mu \mathrm{M}$ (Biasi et al. 1998) and in Vitis vinifera $\mathrm{cv}$. Merlot, in which 2.5 and $5 \mu \mathrm{M}$ BAP favored the calli formation in the internodes, reaching 56.6 and $61.6 \%$, respectively (Carvalho et al. 2011). However, it is difficult to induce efficiently the shoots in the grapevines using the BAP without inducing the calli simultaneously. When the purpose is to produce in vitro seedlings, direct organogenesis (i.e., without an intermediate callus phase) is preferred in order to avoid the somaclonal variation. On the other hand, somaclonal variation can be useful source of new variation for genetic breeding.

The diversity of results obtained for both the cultivars applying the same culture medium, BAP levels, carbon source and amino acids indicated that the in vitro development was genotypedependent.

\section{CONCLUSIONS}

For in vitro shoot regeneration from the nodal segments, best results culture on solid medium were with $5 \mu \mathrm{M}$ BAP, 15 g. $\mathrm{L}^{-1}$ sucrose for the Bordô and 45 g.. $\mathrm{L}^{-1}$ sucrose for the Chardonnay, supplementation with 40 g. $\mathrm{L}^{-1}$ arginine for the 
Bordô and 40 g. $\mathrm{L}^{-1}$ arginine or glycine for the Chardonnay.

\section{REFERENCES}

Abdoli M, Moieni A, Dehghani H. Effects of cultivar and agar concentration on in vitro shoot organogenesis and hyperhydricity in sunflower (Helianthus annuus L.). Pak. J. Bot. 2007; 39: 31-35.

Alberts B, Johnson A, Lewis J, Raff M, Roberts K, Walter P. Molecular Biology of the Cell. 4th ed. London: Garland Science; 2002.

Ayub RA, Spinardi B, Basso MF, Biasi LA. Indução de multibrotação in vitro em videira cv. Bordô. Rev. Bras. Frutic. 2010; 32: 675-681.

Basso MF, Fajardo TVM, Eiras M, Ayub RA, Nickel O. Detecção e identificação molecular de vírus associados a videiras sintomáticas e assintomáticas. Cienc. Rural. 2010; 40: 2249-2255.

Behrend J, Mateles RI. Nitrogen metabolism in plant cell suspension cultures. II. Role of inorganic acids during growth on ammonia. Plant Physiol. 1975; 58: 510-512.

Biasi LA, Passos IRS, Pommer CV. Estabelecimento in vitro de porta-enxertos de videira através de ápices meristemáticos e segmentos nodais. Sci. Agric. 1998; 55: 196-202.

Botelho RV, Pires EJP, Moura MF, Terra MM, Tecchio MA. Garlic extract improves budbreak of the 'Niagara Rosada' grapevines on sub-tropical regions. Cienc. Rural. 2010; 40: 2282-2287.

Carvalho DC, Biasi LA, Telles CA. Organogênese do caquizeiro 'Fuyu' a partir de ápices meristemáticos. R. Bras. Agrociência. 2004; 10: 303-307.

Carvalho DC, Silva ALL, Tanno GN, Purcino M, Biasi LA. Organogenesis from leaf segments and internodes of grapevine cv. Merlot. Ciênc Agrotec. 2011; 35: 108-114.

Corrêa LR, Paim DC, Schwambach J, Fett-Neto AG. Carbohydrates as regulatory factors on the rooting of Eucalyptus saligna Smith and Eucalyptus globulus Labill. Plant Growth Regul. 2005; 45: 63-73.

Couselo JL, Varela P, Rey M. Effect of benzyladenine concentration and double-phase culture system on in vitro multiplication of adult Albariño plants. Am J Ento Vitic. 2006; 57: 109-112.

Cuenca B, Vieitez AM. Influence of carbon source on shoot multiplication and adventitious bud regeneration in in vitro beech cultures. Plant Growth Regul. 2000; 32: 1-12.

Cruz CD. Programa genes: versão Windows: aplicativo computacional em genética e estatística. Viçosa: UFV; 2001.
Eapen S, George L. Plant regeneration from leaf discs of peanut and pigeonpea: Influence of benzyladenine, indoleacetic acid and indoleacetic acid-amino acid conjugates. Plant Cell Tiss Organ Cult. 1993; 35: 223-7.

Goussard PG. Effects of cytokinins on elongation, proliferation and total mass of shoots derived from shoot apices of grapevine cultured in vitro. Vitis. 1981; 20: 228-234.

Haq I, Dahot MU. Effect of permanent and temporary immersion systems on banana micropropagation. Pak. J. Bot. 2007; 39: 1763-1772.

Jaskani MJ, Abbas H, Sultana R, Khan MM, Qasim M, Khan IA. Effect of growth hormones on micropropagation of Vitis vinifera L. cv. Perlette. Pak. J. Bot. 2008; 40: 105-109.

Karasawa MMG, Pinto JEBP, Pinto JC, Pereira AV. Proliferação de capim elefante em diferentes concentrações de regulador de crescimento e consistências do meio de cultura. Ciênc Agrotec. 2002; 26: 1243-1251.

Kirby EG, Leustek T, Lee MS. Nitrogen nutrition. In: Bonga JM, Durzan DJ, editors. Cell and tissue culture in forestry I. General principles and biotechnology. Dordrecht: Martinus Nijhoff Publishers; 1987. p. 6788.

Kozai T, Kubota C, Jeong BR. Environmental control for the large-scale production of plants through in vitro techniques. Plant Cell Tiss Organ Cult. 1997; 51: 49-56.

Machado MP, Biasi LA, Ritter M, Ribas LLF, Koehler HS, Zanette F. Meios de cultura na micropropagação do porta-enxerto de videira "VR043-43" (Vitis vinifera x Vitis rotundifolia). Cienc. Rural. 2007; 37: 277-280.

Murashige T, Skoog F. Revised medium for rapid growth and bioassays with tobacco tissue culture. Physiol Plant. 1962; 15: 473-497.

Oliveira Y, Pinto F, Silva ALL, Guedes I, Biasi LA, Quoirin M. An efficient protocol for micropropagation of Melaleuca alternifolia Cheel. In Vitro Cell Dev Biol Plant. 2010; 46: 192-197.

Palavan-Unsal N, Arisan D. Nitric oxide signalling in plants. Bot. Rev. 2009; 75: 203-229.

Passos IRS, Sondahl MR, Ribeiro IJA, Terra MM, Pires EJP. Cultura in vitro de meristemas de videira: I., concentrações do hormônio 6-BA em meio primário. Bragantia. 1985; 44: 473-479.

Polacco JC. Nitrogen metabolism in soybean tissue culture. Plant Physiol. 1977; 59: 827-830.

Radmann EB, Bianchim VJ, Fachinello JC, Ferreira $\mathrm{LV}$, Oliveira RP. In vitro multiplication of 'Flordaguard' rootstock: cytokinin source and concentration effects, explants orientation and period of permanence in the culture medium. Braz. Arch. Biol. Technol. 2010; 54: 25-34. 
Robinson J. The Oxford Companion to Wine. Oxford: University Press; 1999.

Rodrigues PS, Lindsey GG, Fernandes BMP. Biotechnological approaches for plant viruses resistance: From general to the modern RNA silencing pathway. Braz. Arch. Biol. Technol. 2009; 52: 795-808.

Rossetti MLR. Célula e seus constituintes moleculares. In: Zah A, editor. Biologia molecular básica. Porto Alegre: Mercado Aberto; 1996. p. 13-35.

Rossetto M, Dixon KW, Bunn E. Aeration: a simple method to control vitrification and improve in vitro culture of rare Australian plants. In Vitro Cell Dev Biol Plant. 1992; 28: 192-196.

Scheidt GN, Arakaki AH, Chimilovski JS, Portella ACF, Spier MR, Woiciechowski AL, et al. Utilization of the biorreactor of immersion by bubbles at the micropropagation of Ananas comosus L. Merril. Braz. Arch. Biol. Technol. 2009; 52: 37-43.

Scheidt GN, Silva ALL, Oliveira Y, Costa JL, Biasi LA, Soccol CR. In vitro growth of Melaleuca alternifolia Cheel in bioreactor of immersion by bubbles. Pak. J. Bot. 2011; 43(6): 2937-2939.

Schuck MR, Lipski B, Silva ALL, Carvalho DC, Biasi LA. Acclimatization of micropropagated plants of fox grape cv. Bordô (Vitis labrusca L.) in different substrates. J. Biotec. Biodivers. 2012; 3(4): 206-212.
Silva ALL, Costa JL, Alcantara GB, Carvalho DC, Schuck MR, Biasi LA, et al. Micropropagation of Nidularium innocentii Lem. and Nidularium procerum Lindm. (Bromeliaceae). Pak. J. Bot. 2012; 44(3): 1095-1101.

Taiz L, Zeiger E. Fisiologia Vegetal. Porto Alegre: Artmed; 2004.

Tang W, Newton RJ, Outhavong V. Exogenously added polyamines recover browning tissues into normal callus cultures and improve plant regeneration in pine. Physiol Plant. 2004; 122: 386-395.

Tiburcio AF, Kaur-Sawhney P, Ingersoll RB, Galston AW. Relation between polyamines and pyrrolidine alkaloids in developing tobacco callus. Plant Physiol. 1985; 78: 323-326.

Ziv M. Enhanced shoot and cormlet proliferation in liquid cultured gladiolus buds by growth retardants. Plant Cell Tiss Organ Cult. 1989; 17: 101-110.

Received: August 08, 2011; Revised: December 19, 2011; Accepted: September 25, 2012. 


\section{PAGINA EM BRANCO}

